\title{
Safety of Perioperative Anticoagulant Discontinuation in Cardiovascular Patients Undergoing Periodontal Therapy
}

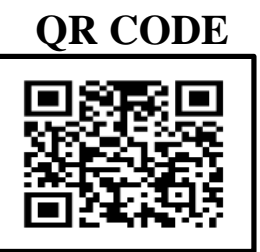

\author{
RITESH VATSA*1, PRIYANKA PRIYADARSHNI²
}

Periodontists are encountering increasing number of cases in their practices where patients present with cardiovascular diseases. Patients with a history of myocardial infarction, ischaemic stroke, acute coronary syndrome or peripheral vascular disease are prescribed anticoagulant medications by cardiologists, such as aspirin, warfarin, clopidogrel, ticlopidine, prasugrel, dipyridamole, cilostazol or dabigatran- either as monotherapy or dual therapy, for prevention of further thromboembolic events during lifetime. As because these agents act by inhibiting platelet aggregation, they tend to prolong bleeding time. Concerns regarding excessive periprocedural bleeding in patients receiving such drugs have led to some clinicians recommending periprocedural discontinuation of anticoagulant therapy. However, several studies, including systematic reviews and meta-analyses, have proved that there is no significantly increased risk of bleeding associated with continuing anticoagulant therapy when compared with discontinuation or modification of the anticoagulant regimen. In fact, without the anticoagulant medication, these patients have been found to be at increased risk of thromboembolic events with potentially serious consequences. The accepted recommendation, therefore, is that the anticoagulant drug regimens should not be altered or discontinued prior to periodontal treatment procedures, as the risks associated with such discontinuation far outweigh the consequences of prolonged bleeding, which can be well controlled with local measures.

KEYWORDS: Cardiovascular disease, Anticoagulants, Aspirin, Clopidogrel, Bleeding time

\section{INTRODUCTION}

Anticoagulant medications such as aspirin, warfarin, clopidogrel, ticlopidine, prasugrel, dipyridamole, cilostazol and dabigatran are used for the prevention of atherothrombotic events in patients with a history of myocardial infarction, ischaemic stroke, acute coronary syndrome or peripheral arterial diseases, particularly in those who have undergone stent placement following percutaneous coronary intervention, coronary artery bypass surgery, or prosthetic valve replantation. ${ }^{1}$ All anticoagulant medications affect clotting by irreversibly inhibiting platelet aggregation within one (e.g., aspirin, prasugrel) to two (e.g., clopidogrel) hours of administration, with the inhibition lasting for the entire lifespan of the platelets (i.e., 7-10 days). ${ }^{2}$ This anticoagulant effect can only be overcome by the production of new platelets. ${ }^{3}$ Complete recovery of platelet function occurs by day four in $80 \%$ of the cases. ${ }^{4}$ In contrast, non-steroidal anti-inflammatory drugs (NSAIDs) other than aspirin (e.g., ibuprofen, diclofenac, piroxicam etc.) have a reversible effect on platelet aggregation, and normal platelet function is restored once the drug is cleared from the circulatory system. ${ }^{3}$ Thus, NSAIDs other than aspirin are not used clinically for anticoagulant therapy.

All patients receiving anticoagulant therapy are likely to possess drug-induced altered platelet function. ${ }^{5}$ Low-dose aspirin can double the baseline bleeding time while clopidogrel may prolong it by 1.5 to 3 times; however, the resulting bleeding time may still be within the normal limits. ${ }^{3,6}$ In a placebocontrolled study, designed to assess the effect of cessation of low-dose aspirin on bleeding time in healthy volunteers after 14 days of treatment, the mean prolongation of bleeding time after 24 hours of cessation of treatment was 22 secs, 105 secs, and 211 secs, respectively, for patients on placebo, $75 \mathrm{mg}$ aspirin, and $300 \mathrm{mg}$ aspirin (normal range of bleeding time specified in the study: $120-600$ secs). ${ }^{7}$ Bleeding times in all the subjects returned to baseline values by the sixth day. Although the effect on primary hemostasis is marginal when anticoagulants are used as monotherapy, ${ }^{8}$ there is likely to be an increased bleeding tendency if they are used as a combination therapy. ${ }^{6}$

\section{Risk Associated with Perioperative Anticoagulant Discontinuation}

Because of concerns about excessive periprocedural and postprocedural bleeding, many clinicians recommend reducing the dosage or completely stopping anticoagulant medications prior to carrying out periodontal surgical therapy and even subgingival scaling and root surface debridement. However, evidence from the extant literature and current consensus indicate that anticoagulant drug regimens should not be altered prior to dental surgical procedures..$^{9-13}$ Stroke and myocardial infarction have been increasingly associated with 
perioperative interruption or cessation of anticoagulant drug regimen. In a prospective cohort study of 1358 hospitalized patients with acute coronary syndrome, $6 \%(\mathrm{n}=73)$ had recently (within 12 days prior to admission) discontinued oral anticoagulants for planned surgery. ${ }^{14}$ Many of these patients had suffered catastrophic outcomes during the 30-day follow-up period with a two-fold increase in mortality rate and major bleeding episodes when compared with those who did not discontinue. In a similar study of 1236 patients admitted to the hospital for acute coronary syndrome, it was found that $4 \%$ of the patients $(n=51)$ had stopped aspirin in the month prior to admission. ${ }^{4}$ However, $13.3 \%$ of all recurrences were from this group, with the mean $( \pm$ SD) delay between aspirin withdrawal and the acute coronary event being $10( \pm 1.9)$ days (range: 4-17 days). Of these, 13 patients $(25.5 \%)$ discontinued aspirin prior to receiving periodontal treatment and dental extractions. In a retrospective study involving 475 hospitalized patients with myocardial infarction, one patient, who had been stable and symptom free on aspirin for 10 years, discontinued aspirin therapy prior to a planned periodontal surgical procedure and suffered a myocardial infarction 10 days after discontinuing aspirin therapy. ${ }^{15}$

A meta-analysis of studies reporting on the cardiovascular risks associated with the continued use of low-dose aspirin versus its perioperative discontinuation, found that out of 93 patients presenting with acute vascular events after cessation of low-dose aspirin, 14 (15.1\%) had discontinued due to planned dental surgery. ${ }^{16}$ The time interval between aspirin discontinuation and acute vascular event was $8.5 \quad( \pm 3.6)$ days for acute coronary syndrome, 14.3 ( \pm 11.3$)$ days for acute cerebral events, and $25.8( \pm 18.1)$ days for acute peripheral vascular events. These observations reinforce the belief that rebound hypercoagulability may occur following abrupt cessation of anticoagulant medication resulting in an increased cardiovascular risk of about 11 events per 1000 patients per week (the background cardiovascular event rate being about 1.4/1000 patients/week). ${ }^{16}$ Patients with coronary stents are at high risk of thromboembolic events and are usually prescribed dual anticoagulant therapy for the prevention of such events in future.

In these patients, it has been observed that the greatest risk for stent thrombosis after implantation is premature discontinuation of clopidogrel. A systematic review examining the effects of short- term discontinuation of anticoagulant therapy in patients with drug-eluting stents found that out of the 161 cases of late stent thrombosis (occurring $\geq 30$ days after insertion), 142 (88\%) had stopped one or both of their anticoagulant medications perioperatively. ${ }^{17}$ In 33 patients who discontinued both anticoagulants simultaneously, the median time to event was 7 days (39\% occurred within 5 days of cessation). In 15 patients who stopped aspirin after having previously discontinued clopidogrel, the median time to event was also 7 days (27\% occurred within 5 days). Moreover, in 94 patients who stopped clopidogrel but remained on aspirin, the median time to event was 122 days ( $2 \%$ within 5 days). There were no events in patients who stopped aspirin but continued with clopidogrel.

\section{Risk of Bleeding Associated with Continuation of Anticoagulant Medications}

Patients taking oral anticoagulants have a prolonged bleeding time; however, this may not be clinically relevant. Clinically significant post-operative bleeding following dental procedures has been defined as bleeding that continues beyond 12 hours, causes the patient to return to the dental clinic or to an emergency department, results in the development of a large haematoma or ecchymosis within the oral soft tissues, or requires a blood transfusion. ${ }^{18}$ When compared with some other types of systemic surgical procedures, routine periodontal therapeutic procedures and minor dental surgeries carried out in the clinic setting, are less likely to be affected by prolonged bleeding time. ${ }^{19,20}$ Blood loss is usually minimal and has minor consequences for the patient even if prolonged. Furthermore, local hemostatic measures can be easily applied in the oral cavity. Thus, for most dental procedures, any increased risk of bleeding does not necessarily increase clinically significant adverse outcomes for the patient or affect the result of the procedure. ${ }^{1}$ In a randomized placebocontrolled study involving 36 healthy patients receiving either aspirin $325 \mathrm{mg}$ or placebo for two days prior to periodontal therapy like flap curettage and two days post treatment, no significant difference between aspirin and placebo was found with respect to intra-oral bleeding time (7.2 $[ \pm 5.9]$ and $5.9[ \pm 6.2] \mathrm{min}$, respectively; $\mathrm{P}=0.51)$ or the duration of bleeding following flap curettage (3.5 $[ \pm 2.9]$ and $5.2[ \pm 6.3]$ hrs, respectively; $\mathrm{P}=0.31) .{ }^{21}$

In another prospective study comprising 82 patients undergoing open flap debridement, the mean 
bleeding time for those who stopped their regular low-dose aspirin before surgery $(n=25)$ was not clinically or statistically significantly increased when compared with those who continued aspirin therapy without any interruption or alteration $(n=32)$ and those who had never taken anticoagulant medication ( $3 \mathrm{~min}[ \pm 2 \mathrm{~min} 45 \mathrm{sec}$ ] vs. $2 \mathrm{~min} 45 \mathrm{sec}$ [ \pm $1 \mathrm{~min} 38 \mathrm{sec}]$ and $1 \mathrm{~min} 49 \mathrm{sec}\left[\begin{array}{lll} \pm & 39 & \mathrm{sec}\end{array}\right]$, respectively). ${ }^{22}$ No patient experienced any episode of prolonged or significant bleeding post-surgery. In another study conducted on 39 patients undergoing dental extractions, none of the patients who continued with aspirin as prescribed $(\mathrm{n}=19)$ had a bleeding time outside normal range. ${ }^{23}$ Intraoperative bleeding was controlled in $85 \%$ of the patients $(\mathrm{n}=33)$ with gauze packing and proper suturing. The remaining 6 patients had tranexamic acid added to the gauze pack. No patient experienced uncontrolled bleeding in the immediate postoperative period or in the week following the procedure. In contrast to anticoagulant monotherapy, fewer studies are available on the bleeding risks of dual anticoagulant therapy. Reports suggest that the use of aspirin in combination with dipyridamole does not increase bleeding risk. ${ }^{24}$ In a retrospective cohort study involving 43 patients on single $(n=14)$ or dual $(n=29)$ anticoagulant therapy (aspirin+ clopidogrel, $\mathrm{n}=\mathbf{2 6}$; aspirin+dipyridamole, $\mathrm{n}=2$; aspirin+ticlopidine, $\mathrm{n}=1$ ) who had received at least one invasive dental procedure, no episodes of postoperative bleeding complications were observed in the 213 dental extractions (including 19 surgical extractions) performed. ${ }^{25}$ Although published studies on the relative risks of perioperative bleeding with clopidogrel or dipyridamole monotherapy are limited, the pharmacological mechanisms underlying their anticoagulant action suggest that patients taking these medications as monotherapy will be at no greater risk of bleeding than those taking aspirin. Therefore, patients on either clopidogrel or dipyridamole monotherapy should not have their therapy discontinued or altered prior to undertaking periodontal surgical procedures. ${ }^{26}$ NSAIDs other than aspirin may also increase bleeding time; however, this seldom exceeds normal limits. Even major surgery is usually not complicated by these drugs, and therefore, they should not be discontinued prior to undertaking periodontal therapeutic procedures. ${ }^{27}$

\section{Weighing the Risk of Thromboembolic Events with that of Periprocedural Bleeding}

Risk of thromboembolic complications, including fatalities, associated with perioperative cessation of anticoagulant monotherapy is well documented. Although the risk is relatively low, the adverse outcome is potentially serious. On the contrary, not a single report exists of uncontrollable bleeding following dental or periodontal treatment undertaken without anticoagulant discontinuation. The meta analysis carried out by Burger and colleagues $^{16}$ examining the cardiovascular risks associated with perioperative discontinuation of low-dose aspirin therapy prior to surgical procedures versus the bleeding risks associated with the continuation of therapy found that surgeons who were blinded to treatment failed to distinguish between patients taking aspirin and those discontinues it. In most cases, bleeding complications were handled in the same way as they would be without the effect of aspirin. The study also showed that discontinuation of aspirin resulted in serious adverse consequences such as stroke, myocardial infarction, and cardiovascular death. In the light of available evidence, guidance documents have been published advising that anticoagulant therapy should only be discontinued in the perioperative period when the haemorrhagic risk of continuing them is categorically higher than the cardiovascular risk associated with their discontinuation. ${ }^{5}$ Furthermore, since the premature interruption of clopidogrel or aspirin plus clopidogrel carries the risk of major adverse cardiovascular events that have devastating consequences, patients taking dual anticoagulant therapy should never have their anticoagulant regimen altered without the advice of an interventional cardiologist. ${ }^{1}$

The American Dental Association, American Heart Association, American College of Cardiology, American College of Surgeons, and Society for Cardiovascular Angiography and Interventions have issued a joint advisory statement specifying the importance of preventing premature discontinuation of dual anticoagulant therapy in patients with coronary artery stents.[28] It states that there is little or no indication to interrupt anticoagulant drug regimen for dental procedures. 


\section{Managing the Risk of Bleeding in Patients Receiving Anticoagulant Therapy}

Dental procedures that can be undertaken in the clinic, including nonsurgical extraction of upto three teeth, surgical removal of teeth, gingivectomy, periodontal flap surgery, apicoectomy, scaling and root planing, etc., can be safely carried out without altering or interrupting anticoagulant medication. ${ }^{18}$ Such patients should ideally be scheduled at the beginning of the day, preferably at the beginning of the week. Local anaesthetic containing a vasoconstrictor should ideally be used in these patients. ${ }^{18,29}$ Regional nerve blocks should be avoided wherever possible. In case of tooth extraction, the extraction sockets should be cleaned and gently packed with a resorbable hemostatic dressing, such as oxidised cellulose, collagen sponge, or resorbable gelatin sponge and carefully sutured..$^{30}$ Resorbable sutures are preferred as they attract less plaque accumulation..$^{30}$ For the management of postoperative pain, paracetamol is a safe overthecounter analgesic in these patients and can be taken in normal doses for pain control. Additional options include dihydrocodeine. Other NSAIDs are considered less safe and should be avoided if possible. They may damage the gut lining leading to bleeding and ulceration, which may be further worsened by aspirin or clopidogrel administration. Some patients under anticoagulant medications may have additional medical problems, such as liver or renal impairment, thrombocytopenia, haemophilia, or other coagulation disorders, which might compound or increase the risk of prolonged bleeding after dental extractions and/or periodontal surgeries. ${ }^{1}$ Before treating these patients, the dentist must consult with the patient's physician or haematologist to determine whether or not care can be safely delivered in the dental clinic.

\section{CONCLUSION}

Bleeding complications, while troublesome, do not carry the same risks as thromboembolic complications. Bleeding time in patients receiving anticoagulant medications is likely to be prolonged; however, perioperative or postoperative blood loss in these patients is usually minimal and should be manageable using local haemostatic measures only. These patients are placed at an increased risk of severe thromboembolic events with potentially serious consequences if their regular anticoagulant medications are discontinued. The recommendation therefore is that the anticoagulant drug regimen should never be stopped or altered prior to any dental surgical procedure, which includes periodontal surgical therapy as well as exodontia.

\section{Conflict Of Interest \& Source of Funding}

The author declares that there is no source of funding and there is no conflict of interest among all authors.

\section{REFERENCES}

1. Surgical management of the primary care dental patient on antiplatelet medication. North West Medicines Information Centre. Liverpool, UK. August 2010.

2. Merritt JC, Bhatt DL. The efficacy and safety of perioperative antiplatelet therapy. J Thromb Thrombolysis 2002;13:97-103.

3. Schafer AI. Effects of nonsteroidal antiinflammatory drugs on platelet function and systemic haemostasis. J Clin Pharmacol 1995;35:20919.

4. Ferrari E, Benhamou M, Cerboni P, Marcel B. Coronary syndromes following aspirin withdrawal. A special risk for late stent thrombosis. J Am Coll Cardiol 2005;45:456-9.

5. Samama CM, Bastien O, Forestier F, Denninger $\mathrm{MH}$, Isetta c, Juliard JM, et al. French Society of Anaesthesiology and Intensive Care. Antiplatelet agents in perioperative period: expert recommendations of the French Society of Anaesthesiology and Intensive Care (SFAR) 2001summary statement. Can J Anaesth 2002;49:S26-35. 6. Lecompte T, Hardy JF. Antiplatelet agents and perioperative bleeding. Can J Anaesth 2006;53:S10312.

7. Cahill RA, McGreal GT, Crowe BH, Ryan DA, Manning BJ, Cahill MR, et al. Duration of increased bleeding tendency after cessation of aspirin therapy. J Am Coll Surg 2005;200:564-73.

8. Mielk CH Jr. Aspirin prolongation of the template bleeding time: Influence of venostasis and direction of incision. Blood 1982;60:1139-42.

9. Douketis JD, Berger PB, Dunn AS, Jaffer AK, Spyropoulos AC, Becker RC, et al. American College of Chest Physicians. The perioperative management of antithrombotic therapy; America College of Chest

Physicians Evidence-Based Clinical Practice Guidelines $\quad\left(8^{\text {th }} \quad\right.$ edition $)$ Chest $2008 ; 133(6$ Suppl):299S-339S.

10. Sandor G. Do patients taking oral anticoagulants need to discontinue their medication before surgical procedures? J Can Dental Assoc 2004;70:482-3. 
11. Nematullah A, Alabousi A, Blanas N, Douketis JD, Sutherland SE. Dental surgery for patients on anticoagulant therapy with warfarin: a systematic review and meta-analysis. J Can Dent Assoc 2009;75:41a-i.

12. Perry DJ, Noakes TJ, Helliwel PS. British Dental Society. Guidelines for the management of patients on oral anticoagulants requiring dental surgery. $\mathrm{Br}$ Dent J 2007;207:389-93.

13. Baker RI, Coughlin PB, Gallus AS, Harper PL, Salem HH, Wood EM. Warfarin Reversal Consensus Group. Warfarin reversal: consensus guidelines, on behalf of the Australian Society of Thrombosis and Haemostasis. Med J Aust 2004;181:492-7.

14. Collet JP, Montalescot G, Blanchet B, Tanguy ML, Golmard JL, Choussat R, et al. Impact of prior use or recent withdrawal of oral antiplatelet agents on acute coronary syndromes. Circulation 2004;110:2361-7.

15. Collet JP, Himbert D, Steg PG. Myocardial infarction after aspirin cessation in stable coronary artery disease patients. Int J Cardiol 2000;76:257-8.

16. Burger W, Chemnitius JM, Kneissel GD, Rucker G. Low-dose aspirin for secondary cardiovascular prevention-cardiovascular risks after its perioperative withdrawal versus bleeding risks with its continuation-review and meta-analysis. J Intern Med 2005;257:399-414.

17. Eisenberg MJ, Richard PR, Libersan D, Filion KB. Safety of short term discontinuation of antiplatelet therapy in patients with drug-eluting stents. Circulation 2009;119:1634-42.

18. Lockhart PB, Gibson J, Pond SH, Leitch J. Dental management considerations for the patient with an acquired coagulopathy. Part 1: Coagulopathies from systemic disease. Br Dent J 2003;195:439- 45.

19. Servin F. Low-dose aspirin and clopidogrel: how to act in patients scheduled for day surgery. Curr Opin Anaesthesiol 2007;20:531-34.

20. Partridge CG, Campbell JH, Alvarado F. The effect of platelet-altering medications on bleeding from minor oral surgery procedures. J Oral Maxillofac Surg 2008;66:93-97.

21. Brennan MT, Valerin MA, Noll JL, Napenas JJ, Kent ML, Fox PC, et al. Aspirin use and postoperative bleeding from dental extractions. J Dent Res 2008;87:740-44.

22. Krishnan B, Shenoy NA, Alexander M. Exodontia and antiplatelet therapy. J Oral Maxillofac Surg 2008;66:2063-6.

23. Ardekian L, Gasper R, Peled M, Brener B, Laufer

D. Does low-dose aspirin therapy complicate oral surgical procedures? J Am Dent Assoc 2000;131:331-5.
24. Persantin/Persantin Retard [summary of product characteristics]. Berkshire, UK: Boehringer Ingelheim Limited;2012.

25. Napenas JJ, Hong CH, Brennan MT, Furney SL, Fox PC, Lockhart PB. The frequency of bleeding complications after invasive dental treatment in patients receiving single or dual antiplatelet therapy. JADA 2009;140:690-5.

26. Joint Formulary Committee. British National Formulary. 59 ed. London, UK: Royal Pharmaceutical Society of Great Britain/British Medical Association; 2010:23.

27. Schafer AI. Effects of non-steroidal antiinflammatory drugs on platelet function and systemic hemostasis. J Clin Pharmacol 1995;35:20919.

28. Grines CL, Bonow RO, Casey DE Jr, Gardner TJ, Lockhart PB, Moliterno DJ, et al. American Heart Association; American Colege of Cardiology; Society for Cardiovascular Angiography and Interventions; American College of Surgeons; American Dental Association; American College of Physicians. Prevention of premature discontinuation of dual antiplatelet therapy in patients with patients with coronary artery stents: a science advisory from the American Heart Association, American College of Cardiology, Society for Cardiovascular Angiography and Interventions, American College of Surgeons, and American Dental Association, with representation from the American College of Physicians. Circulation 2007;115:813-8.

29. Alexander RE. Eleven myths of dentoalveolar surgery. J Am Dent Assoc 1998;129:1271-9.

30. Scully C, Wolff A. Oral surgery in patients on anticoagulant therapy. Oral Surg Oral Med Oral Pathol Oral Radiol Endod 2002;94:57-64. 
Source of support: Nil, Conflict of interest: None declared

Cite this article as:

Vatsa R, Priyadarshini P. Safety of Perioperative Anticoagulant Discontinuation in Cardiovascular Patients Undergoing Periodontal Therapy. Int Healthc Res J. 2018;2(9):207-212. doi: 10.26440/ihrj.v2i9.178

\section{AUTHOR AFFILIATIONS:}

1. MDS (Oral and Maxillofacial Surgery), Senior Resident, Department of Dentistry, Darbhanga Medical College and Hospital, Darbhanga

2. MDS (Prosthodontics), Tutor, Patna Dental College and Hospital, Patna

\section{${ }^{*}$ Corresponding Author:}

Dr. Ritesh Vatsa

Senior Resident

Department of Dentistry

Darbhanga Medical College and Hospital

Darbhanga 